\title{
Cogntive Consistency Analysis in Adaptive Bio- Metric Authentication System Design
}

\author{
Gahangir Hossain \\ Electrical \& Computer Engineering \\ Indiana University-Purdue \\ University Indianapolis \\ Indianapolis, IN USA
}

\author{
Habibah Khan \\ Instructional Design \& Technology \\ The University of Memphis \\ Memphis, TN USA
}

\author{
Md.Iqbal Hossain \\ Electrical \& Computer Engineering \\ The University of Memphis \\ Memphis, TN USA
}

\begin{abstract}
Cognitive consistency analysis aims to continuously monitor one's perception equilibrium towards successful accomplishment of cognitive task. Opposite to cognitive flexibility analysis - cognitive consistency analysis identifies monotone of perception towards successful interaction process (e.g., biometric authentication) and useful in generation of decision support to assist one in need. This study consider fingertip dynamics (e.g., keystroke, tapping, clicking etc.) to have insights on instantaneous cognitive states and its effects in monotonic advancement towards successful authentication process. Keystroke dynamics and tapping dynamics are analyzed based on response time data. Finally, cognitive consistency and confusion (inconsistency) are computed with Maximal Information Coefficient (MIC) and Maximal Asymmetry Score (MAS), respectively. Our preliminary study indicates that a balance between cognitive consistency and flexibility are needed in successful authentication process. Moreover, adaptive and cognitive interaction system requires in depth analysis of user's cognitive consistency to provide a robust and useful assistance.
\end{abstract}

Keywords-Cognitive authentication; Cognitive consistency; Fingertip dynamics; Maximal Information Coefficient; Bivatiate plot

\section{INTRODUCTION}

With the increase of adaptive interfaces including natural gestures, touch-screen, tactile, speech enable, implicit and tangible interactions; fingertips (keystrokes, mouse click and tapping interfaces) are still dominating since their invention [1]. Users like to perform authentic interaction by a fingertip. Slip of tip might hinder the user in robust interaction and authentication process just because of inflexibility of accessibility and authentication schemes. This becomes more challenging when the users need assistance from the interaction system. Meanwhile, the system strategically requires a robust user authentication, adaptation and automation to tie its users continuously into its loop. Question arises: how to maximize authentic accessibility to benefit user in assistive interaction? The challenging accessibility problem requires a different usability engineering solution than we currently practice. Analysis of end-user's cognitive pattern of interaction and deficiencies may improve the future interface accessibility towards authentic and adaptive accessibility design. More specifically, cognitive approach of authentication may allow some flexibility in user authentication process based on users' past history of success and present consistent interactions (monotone), even though unsuccessful in authentication process.

Cognitive consistency analysis is the fundamental principle in social cognition and important factor in balancing interests through adaptive collaborative system design. The analysis uncovers three key factors that tend toward (cognitive) consistency: perception, emotion, and action. More specifically, the process helps the system to reveal user's intrinsic conscious conflict situation that leads to adaptive behavior.

In an adaptive authentication, cognitive consistency analysis is a critical research process, that aims to (1) identify progressive interests in biometric authentication, (2) develops understanding of inherent meaning, values and motives in cognitive activities, (3) study adaptive interaction trends with connection to current research, (4) construct models of the relations between cognitive capability, personal profile and participants' actions, (5) elucidate the fundamental contradiction which are developing as a result of action based on ideologically frozen understanding, (6) participate in a program to see new ways of the situation, (7) Theoretically ground the principles applied in the analysis.

With the holistic goal of adaptive authentication system development, two specific aims are analyzed through the research. First specific aim is to understand consistent or inconsistent interactions during authentic interaction. The second specific aim is to co-analyze user's past history of successful authentication record with the degree of present cognitive activity to provide an essential decision support in authentication process. This study covers the first aim with two different datasets. Moreover, the conditions with understandings are compared, ideologies are criticized, and immanent possibilities for action are discovered.

The rest of the paper is organized as following: In section 2 , gives brief descriptions on cognitive approaches in fingertip dynamics and authentication with general and mixed frameworks. Section 3, explains the methods used in cognitive consistency analysis process. Exploratory analyses of cognitive effort on some benchmark biometric data sets are shown in section 4. Finally, the research is concluded with findings and future works in section 5 . 


\section{FINGERTIPS DYNAMICS APPROACHES}

Fingertips dynamics includes finger related user interactions including keystroke, key tapping, and mouse clicking. Roman and Vanue [1] reviewed a classification of the state-of-the-art behavioral biometrics related to user's skills, style, preference, knowledge, motor-skills or strategy that users use in their everyday task accomplishment. Fingertips dynamics fall into the behavioral biometrics classification and play an importance role in conjunction to everyday hand related activities. Keystroke dynamics biometric pattern analysis and user modeling are proposed by Killourhy[3]. Subjects typed a strong password and their key pressing response times are recoded to have up key, holding and down key and their combination response analysis. Ahmed and Traore's [2] proposed new biometric fingertips with mouse clicking dynamics, which was improved in Nakkabi et al. [4] in terms of clicking response time. Poh and Tistarelli[9] customized biometric authentication systems by a novel method with discriminative score. Liang et al. [10] proposed a combined analysis of fingertips dynamics with head pose estimation. The real-time fingertip gesture tracking is proposed in Oka et al. [13] which determine an appropriate threshold value for image binarization during initialization by examining the histogram of an image of a user's hand placed open on a desk.

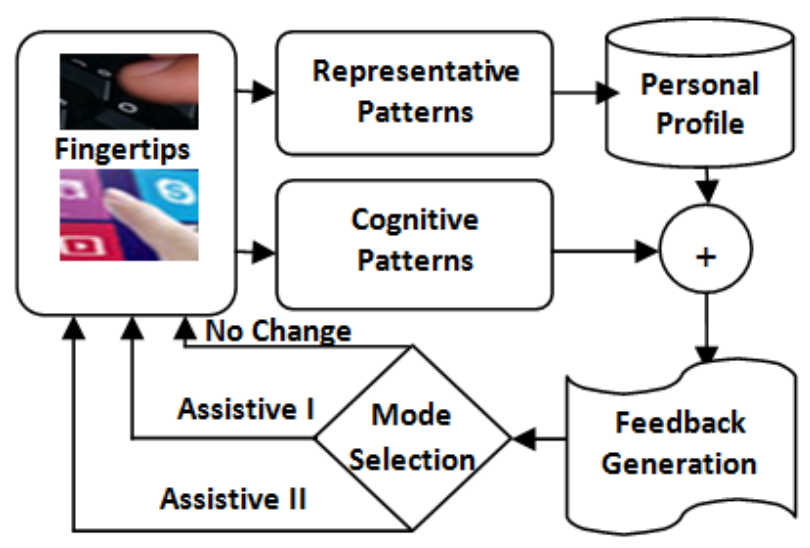

Fig. 1. Overview of proposed cognitive biometric authentication system with flexible feedbacks

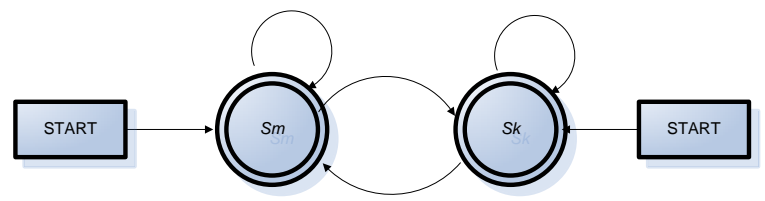

Fig. 2. Keystroke and mouse click are complementary; Sm and Sk stands for the mouse clicking state and keystroke state respectively

Cognitive issues in fingertips includes but not limited to: mental effort, consistencies (monotone) in action, dissonance, bias and more. The cognitive mechanisms of touch are proposed in some works including, Rola [13], Jone et al. [14] gives more elaboration of tactile sensory systems with brain signal analysis. Hossain et al.'s work $[5,6]$ proposes some relevant cognitive mechanism of cognitive workload during assistive technology interaction. These literatures prove an important grounding in cognitive analysis of fingertips dynamics. Fishel[9] proposed a direct measure of fingertip strength through a robust micro-vibration sensor for bio-metric fingertips.

\section{A. The General Structure}

In keystroke and tapping dynamics, user identifiable patterns are considered as representative patterns and recorded as personal profile. Cognitive patterns relates to mental activities which are related to user's task performance in terms of response time in case of fingertip dynamics. For example, the monotone in response times in every key (or dot) a user press (or tap) in authentication process. Combining both type of patterns are proposed in cognitive approach of authentication system. More specifically, the combined approach is considered to be useful in better assistance it's used in need. Figure 1 shows a functional block diagram of cognitive authentication systems with assistive modes. The assistive modes may vary based on flexibility of design principles.

\section{B. The Mixed or Hybrid Structure}

Keystroke and mouse clicking are still considered as primary input mechanisms. While the keystroke is considered as feed-forward interaction, the mouse click is considered as feedback interaction which is mostly replaced by tapping interaction essential in authentication process. These two interaction mechanisms works as two wings of a bird in robust interaction. It is quiet impossible for a user to accomplish a task depending only on key pressing or tapping/mouse interaction rather a combination of keyboard and mouse click/ tapping. Although keystroke and mouse clicks are sometimes complementary, their combination makes the interaction more natural. The main idea of mixed cognitive authentication is to allow the user a natural way of access rights with a combined or individual approach of fingertips and dynamics. Key objectives are: (a) to identify similarity and differences of cognitive efforts a user experiences during continuous fingertips and (b) to analyze the leverage of the differences towards adaptive authentication design. Figure 2 shows a state diagram of cognitive authentication with possible beginning and ending states of keyboard and mouse clicks use in action. Measures of cognitive effort dynamics become important and challenging research issue.

This work analyzed the keystroke response time and fingertips dynamics to have insights of cognitive efforts and their differences.

\section{Theoretical Background}

Naturally, we expect and have a preference for monotonic interaction in our lives as well as other things including in authentication process. We need consistency in the whole interaction (e.g., key pressing) process of authentication. This section explains cognitive consistency analysis with a theory and mathematical modeling techniques.

\section{1) Cognitive consistency theory}

Consistency becomes like a form of human gravity. It holds everything down and together. It helps us to understand the world and our place in it. The fundamental thrust of consistency theories is to enforce equilibrium among one's 
cognitions. Cognitive consistency can be defined with cognitive consistency theory[7 -9], that focuses on the balance individuals create cognitively when inconsistencies create tensions and thus motivate our brains and body to respond. The fundamental thrust of cognitive consistency theory is to enforce equilibrium among one's cognitions. Cognitive consistency theory shows how people motivate themselves to work and adjust inconsistent measures. Three steps of consistency theory are: (1) estimation of expected consistency, (2) resolving inconsistencies that create a state of dissonance, and (3) the dissonance drives us to restore consistency. This theory is the basis for equilibrium for individuals in authentication process. However, the importance of positive and negative outcomes to reduce stressful choices relates to cognitive dissonance theory of Leon Festinger[18, 19]. Although, the cognitive consistency theory touches on both issues, it focuses on the affects of inconsistencies in interaction process motivating to react and consequent actions.

Fingertips dynamics represent finger related user interactions including keystroke, key tapping, and mouse clicking. Cognitive issues in fingertips includes but not limited to: cognitive effort, consistencies (monotone) in action, dissonance, bias and more. For example, consider in keystroke and tapping dynamics. User identifiable patterns are considered as representative patterns and recorded as personal profile. Cognitive patterns are some derived patterns from online task performance in terms of response time in fingertip dynamics. Combining both type of patterns are proposed in cognitive approach of authentication system.

Main research goal is to have an adaptive interface that can understand consistency of mental state in fingertip dynamics. More specifically, the goal is to have a robust and effective user accessibility framework which is useful in flexible user authentication process. Cognitive analysis of fingertips dynamics can be combined with users' prior interaction behavior profiles to have more effective authentication system. Cognitive effort, load, and cost are some analysis considered in consistent authentication interface design.

Figure 3, illustrates an example of lock pattern tapping interaction and cognitive analysis to understand user's consistent and dissonance interactions. Figure 3, a \& b shows the schematic of interaction process. Relative response time between dots or dot-to-dot transition may uncover the cognitive consistence. Main assumption behind the consistency identification is that, user with a balance consistencedissonance value need no assistance from the system in authentication process.

\section{THE CRITICAL ANALYSIS}

This work analyzed two datasets: the keystroke dynamics dataset [3], which is publicly available as a benchmark dataset and the lock pattern tapping [18] dataset - used with permission from the author. Both dataset are passed through the institutional review board (IRB) for secondary evaluation. Maximal Information-based Nonparametric Exploration (MINE) tool [17] is used in consistency and dissonance analysis.

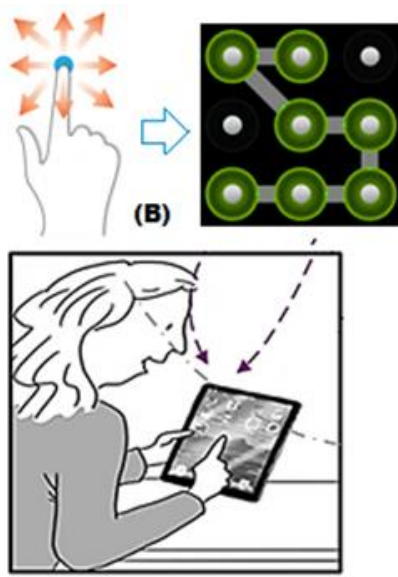

(A)

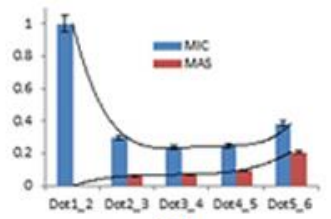

(C)

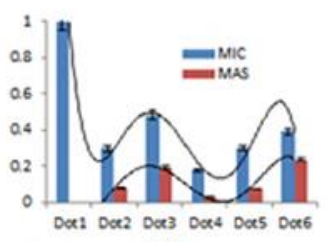

(D)
Fig. 3. Cognitive authentication in lock pattern tapping (fingertips) dynamics analysis

\section{A. Technical Analysis}

The consistency is analyzed with maximal information coefficient (MIC), which is a non-parametric measure of twovariable dependence. The MIC is widely used to identify important relationships in data sets and to characterize them. Different relationship types give rise to characteristic matrices with different properties. For instance, strong relationships yield characteristic matrices with high peaks, monotonic relationships yield symmetric characteristic matrices, and complex relationships yield characteristic matrices whose peaks are far from the origin. The MIC is used to measure relationship strength between two responses (say, dot1 and dot2) in terms of response time. Let the two response variables be defined as D and A, respectively. The MIC can be written as

$$
\begin{aligned}
\operatorname{MIC}(M)=\max _{\mathrm{DA}<\mathrm{B}(\mathrm{n})} & \operatorname{Mutual}(\mathrm{M})_{\mathrm{D}, \mathrm{A}} \\
= & \max _{\mathrm{DA}<\mathrm{B}(\mathrm{n})} \frac{\mathrm{I} *(\mathrm{M}, \mathrm{D}, \mathrm{A})}{\log (\min \mathrm{D}, \mathrm{A})}
\end{aligned}
$$

where $B(n)=n$ is the search-grid size, $I(M, D, A)$ is the maximum mutual information over all grids $\mathrm{D}$-by- $\mathrm{A}$, of the distribution induced by $\mathrm{M}$ on a grid having $\mathrm{D}$ and $\mathrm{A}$ bins.

The Maximum Asymmetry Score (MAS)captures the deviation from monotonicity, and useful for detecting periodic relationships to have idea about cognitive dissonance. It can be defined as:

$$
\operatorname{MAS}(\mathrm{M})=\max _{\mathrm{DA}<\mathrm{B}(\mathrm{n})}\left|\operatorname{Mutual}(\mathrm{M})_{\mathrm{D}, \mathrm{A}}-\right| \operatorname{Mutual}(\mathrm{M})_{\mathrm{A}, \mathrm{D}} \mid
$$

The Maximum Edge Value (MEV) measures the closeness to being a function is defined as:

$$
\operatorname{MEV}(D)=\max _{X Y<B(n)}\left\{M(D)_{X, Y}: X=2^{\sim} \text { or } Y=2\right\} .
$$

Cognitive consistency visualization and analysis:

Box plot and robust bi-variant bag plots are used to visualize fingertips dynamics (consistencies) in terms of data location, spread, skewness and outliers. 


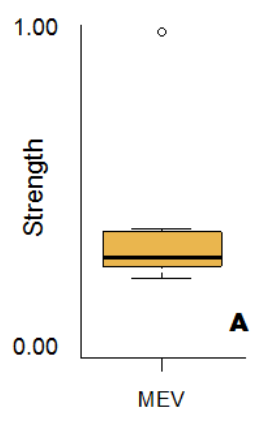

A.

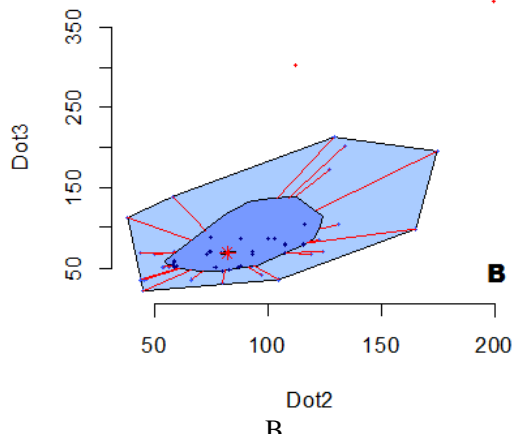

B.
Fig. 4. Bi-variate plots illustration of two consicutive dots responsetime. Left - A. the box plot and Right - B. the bag plot

Boxplot shows the difference in cognitive levels at fingertips inconsistencies and control. Boxes show the median, 25th and 75th percentiles, error bars show 10th and 90th percentiles, and filled symbols show outliers (figure 4A).

In bag plot, the representation consists of three nested polygons: 'bag', 'fence' and 'loop' (figure 4B). The bag is the inner polygon, which is a construction of the smallest depth region containing at least $50 \%$ of the total number of observations, also known as Tukey depth around the center point of median (Tukey median). The most outer polygon known as fence (which is not drawn in the figure) but computed for the outlier points identification. Observations outside the fence are flagged as outliers. The 'fence' polygon can be obtained by inflating the bag (relative to the Tukey median) by a factor $\rho$. According to Rousseeuw et al. [21] $\rho=$ 3 , a recent work [22] prefers $\rho=2.58$, as that allows the fence to contain $99 \%$ of the observations when the projected bivariate scores follow standard normal distributions. The convex hull of the observations that are not marked as outlier creates another polygon known as loop. The highest possible Tukey depth (median) is also marked in orange color near the center of the graph. The importance of use of bagplot is not only it's robustness against outliers, but also invariant under afine transformation.

\section{B. Datasets}

The Keystroke Dynamics - Benchmark Data Set is the accompaniment to Kevin and Roy [3]. The data consist of keystroke-timing information from 51 subjects (typists), each typing a password (.tie5Roanl) 400 times. The second dataset is - the lock pattern tapping dataset [20] - according to [20] a total of 32 different participants successfully completed lock pattern task assigned to them using the mobile application. Participants were 20 male and 12 female with different age groups (from 19 to 56 years old), cultural and educational backgrounds, and having different levels of experience interacting with touch-screen smart phones. They performed the test on different Android phones: Samsung Galaxy SII (18), Nexus S (8), HTC Legend (4) and HTC Vision (2). With email permission from [20], this dataset is used in this study for secondary evaluation.

MIC, MAS and MEV are performed in both dataset to identify underlying monotone, inconsistencies and functional closeness, respectively. Bi-variate box and bag plots are performed visual illustration of cognitive states that shows the trends in monotone and consistencies.

\section{RESULTS AND DISCUSSIONS}

Understanding cognitive states are important in accessible and adaptive system development. Ideas presented through this research are transformative and critical (not qualitative or quantitative) and aim to bring additive flexibilities in adaptive interaction design. Finger tapping is gaining increasing popularity; hence, the MIC-MAS trends are analyzed that is observed in finger tapping dataset. Figure 3 shows partial result of monotonic interaction and dissonance in lock pattern tapping interaction. Figure 3, c \& d illustrates the dot-to-dot and in dot cognitive response time relations, respectively. Figure $3 \mathrm{C}$, shows that the increasing number of transition to remember increases more gap (delay). Result shows partial dot-to-dot and in-dot monotonic interaction and inconsistencies in terms of MIC and MAS. In dot-to-dot transition (Figure 3c), user shows monotonically decreasing relative responses which reaches in a steady state in between dot transitions (dot2-3, dot3-4, dot4-5) which was analyzed on all correct lock pattern response data. Moreover, user has an increase of cognitive inconsistencies (dissonance) with increase of dot-to-dot transition options (dot2-3, dot3-4, dot4-5, and dot5-6). Relative in-dot response time (figure3d) shows a stable pattern of MAS value (dissonance) always lower than consistent dot visit (MIC value), which is similar to dot-to-dot analysis, but no steady trend is observed. So, the dot-to-dot transition MIC-MAS relationship uncovers more cognitive mental aspects in authentication process.

Similar to figure $3 \mathrm{c} \& 3 \mathrm{~d}$, in keystroke data analysis, key up to next key down response time and key holding time MIC MAS are separately computed and plotted in figure 5. The cognitive consistency and inconsistency trends in key up-down and key holding response times are shown in figure5 (up) and (down), respectively. While the tapping interaction shows a frequent sinusoidal trend, keystroke interaction shows a delayed period in sinusoids.

Figure 6, shows the box plot of the complete data set with additional MINE tool, the maximum edge value (MEV) in lock pattern tapping data. According to keystroke dynamics dataset [3], users are instructed to try different log patterns. Their tapping time and performance are logged in terms of response time. This study performed an analysis of correlative activities with MIC and MAS. Box plots in figure6, represent overall analysis on MIC, MAS and MAV values. The DD analysis (the right part of the figure) indicates a consistent responses by test subjects in successfully password typing. In figure 7, holding time $(\mathrm{H})$ and key-up-to-key-down (UD) time analysis shows, inconsistencies in MAS value. Whereas, more inconsistencies (dissonance) are observed in holding time $(\mathrm{H})$, cognitively meaning that subject is more confused in selecting and pressing keys related to password.

Deeper lock-pattern tapping analysis is performed with a comparative tapping response time analysis in within and between dots and their transition. 

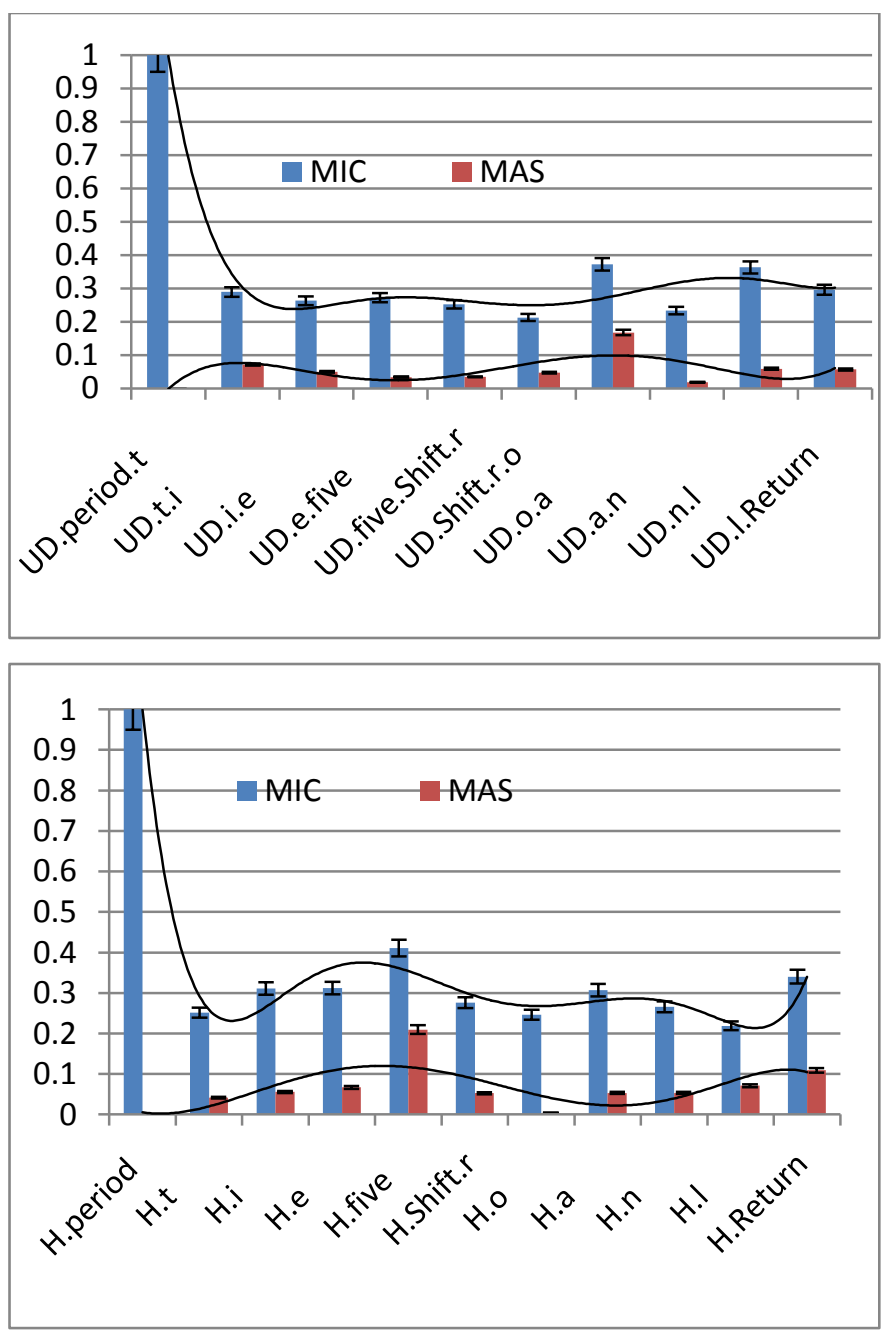

Fig. 5. Trends of user cognitive involvement in keystroke change (top) and key holding (bottom)

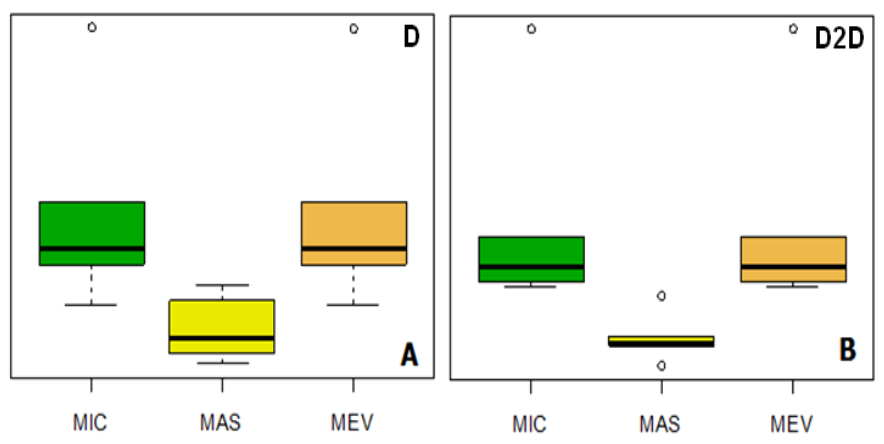

Fig. 6. Box plot of lock pattern tapping data. D in the top right corner of figure A represents the results from the response time analysis on dots. Where D2D represents the response time analysis on dot-to-dot. The Y-axis represents strength in $0.00-1.00$ scale

These are illustrated with bag plots in figure 8. Similarly, in password typing (keystroke dynamics) dataset, inter key holding time and key transition time (key up and key down) and their comparisons are illustrated with bag-plots in figure 9. Both box plots and bi-variate bag plots combined illustrates the underlying cognitive factors in authentication.

Both in, in-dot and key-holding, subjects have similar cognitive states (gray dotted line on box plots figure A, and figure8A), then in dot-to-dot and key-up to key-down time. This observation, illustrates that they have more consistencies in finger tapping memory (recall) task then decision (switching) task. Existence of more sparse outliers, in later bags indicates decision inconsistencies in selecting transitions (C and D parts in figure8 and 9). For instance, the last bag plot of both cases is smaller because of sparse outlier.

Having MIC, MAS and MAV values; and outliers' situation, some derived feature values with keystroke dynamics and finger tapping datasets, a representative feature vector can be created to apply machine learning approaches towards cognitive authentication system design.
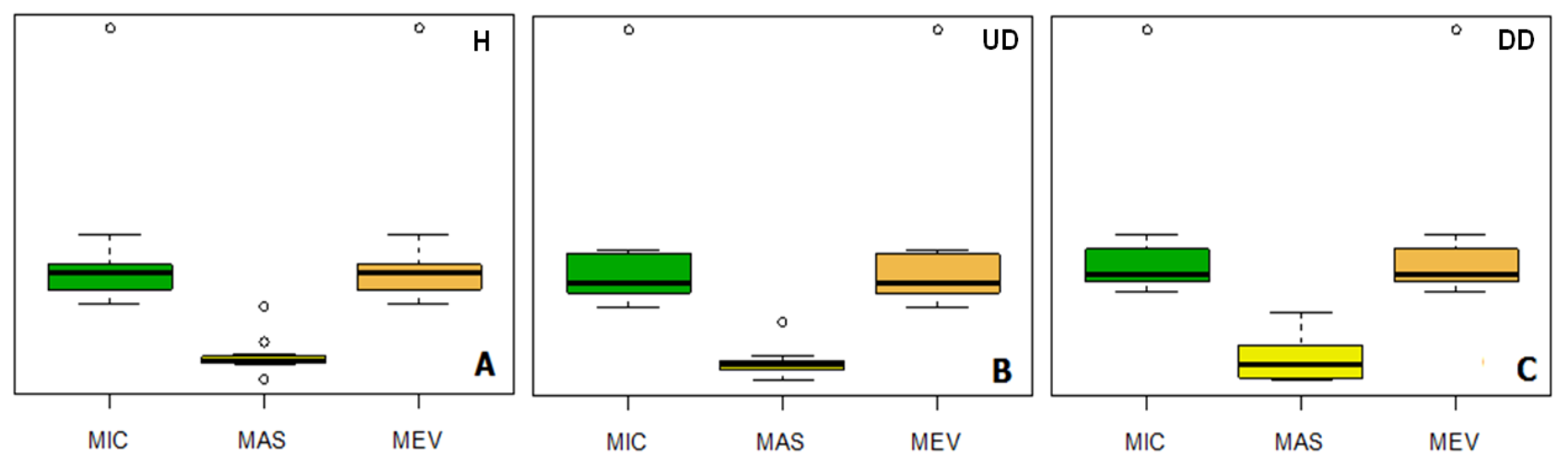

Fig. 7. Box plot of keystroke dynamics dataset[3]. DD in the top right corner in right plot C, represents the results from the key-down to key-down response time analysis. Whereas, $\mathrm{H}$ represents the key holding response time, and UD represents the key-up to key-down response times plot. The Y-axis represents strength in $0.00-1.00$ scale 


\section{CONCLUSION}

Rapid increase of accessible technology needs continuous access of user's ability based authentic interaction - an adaptive-cognitive authentication protocol becomes an important issue. This study started with the old principle of cognitive consistency and inconsistencies (dissonance) theories with novel applications in adaptive authentication system development. Exploratory analyses are performed to identify trends of cognitive activities. Some instances of cognitive processes (perception, attention, and action/decision-making) are considered in this analysis. The associated properties emotion, intuition, collaborative action etc. can be derived with more analysis.

By fingertips dynamics, we can consider tapping, double tapping, long press, scroll, pan, flick, two finger tapping, two finger scroll, pinch, zoom, and rotate during interaction. This paper presents a compilation of user interface requirements that arise in fingertip dynamics based cognitive, assistive and adaptive interaction design. It draws implications imposed by the user interaction requirements on the architectures of cognitive consistent system. It defines a special class of cognitively defined authentication systems. Various cognitive mechanisms of providing efficient mental states can be included with similar framework. This paper also draws attention to the need for more non-parametric analysis in cognitive system design.

This paper provided a way of cognitive consistency analysis in keystroke based user interface requirements for constructing good user experience. A special user interface design needs to have assistive (helps it's user in need) and adaptive (learns and updates personal profile) properties, that can be accomplished through consistency analysis. This analysis presents opportunities to improve the system performance relying on the special structure of such interactions. Some ways to exploit this structure are pointed out through this study. Additional research could be warranted in the following areas to further exploit the nature of such problems. One may experiment with various task outcomes and cognitive load schemas for memorizing passwords and lock patterns of computations across user sessions.

Additional cognitive analysis could be gained by learning across cognitive dissonance theory $[18,19]$. Such learning may involve cognitively confused patterns in test items.

More trial and time could be devoted to devising and experimenting with algorithms that are essential and connected to robust and online accessibility.

\section{REFERENCES}

[1] V. Y. Roman, and G. Venu, "Behavioural biometrics: a survey and classification." International Journal of Biometrics 1, no. 1 pp. 81-113, 2008

[2] A. E. Ahmed and I. Traore, "A New Biometrics Technology based on Mouse Dynamics", IEEE Transactions on Dependable and Secure Computing, Vol. 4 No. 3, pp.165-179, 2007.
[3] K. S. Killourhy, and A. M. Roy, "Comparing anomaly-detection algorithms for keystroke dynamics," Dependable Systems \& Networks, pp.125-134, 2009. IEEE Computer Society Press, Los Alamitos, California, 2009

[4] Y. Nakkabi, I. Traore, A. A. E Ahmed, "Improving Mouse Dynamics Biometric Performance using Variance Reduction with Separate Extractors", IEEE Transactions on Systems, Man and Cybernetics-Part A: Systems and Humans, Vol. 40, No. 6, pp.1345 - 1353,2010.

[5] G. Hossain and M. Yeasin, Cognitive Load Based Adaptive Assistive Technology Design for Reconfigured Mobile Android Phone, MobiCASE 2011, pp.370-380, Los Angeles, CA, USA, 2011.

[6] G. Hossain, A. S. Shaik, and M. Yeasin, Cognitive Load and Usability Analysis of RMAP for people who are blind or visually impaired. In Proceedings of the 29th ACM International Conf. on Design of Communication (SIGDOC) ACM, Pisa, Italy, 2011.

[7] P. Tannenbaum, The Congruity Principles: Retrospective Reflection and Recent Research. In Theories of Cognitive Consistency: A Sourcebook. Abelson, R. \& others (eds.) Rand McNally \& Co.; Skokie, 111, 1968.

[8] M. Venkatesan, Cognitive Consistency and Novelty Seeking. In Ward, S. \& Robertson, T. (eds.) Consumer Behaviour: Theoretical Sources. Prentice-Hall, Inc; Englewood Cliffs, New Jersey, 1973.

[9] T. Newcomb, Interpersonal Balance. In Abelson, R. et al Theory of Cognitive Consistency: A Sourcebook. Rand McNally \& Co. Skokie, 111,1968

[10] N. Poh and M. Tistarelli, "Customizing biometric authentication systems via discriminative score calibration." In Computer Vision and Pattern Recognition (CVPR), 2012 IEEE Conference on, pp. 2681-2686. IEEE, 2012.

[11] H. Liang, Y. Junsong, and D. Thalmann. "Hand pose estimation by combining fingertip tracking and articulated ICP." In Proceedings of the 11th ACM SIGGRAPH International Conference on Virtual-Reality Continuum and its Applications in Industry, pp. 87-90. ACM, 2012.

[12] V. Štruc, Z. Jerneja, V. Boštjan, and N. Pavešić. "Beyond parametric score normalisation in biometric verification systems." IET Biometrics 3, no. 2 (2014): 62-74.

[13] K. Oka, S. Yoichi, and K. Hideki, "Real-time fingertip tracking and gesture recognition." Computer Graphics and Applications, IEEE 22, no. 6 (2002): 64-71.

[14] E. T. Rolls, "The affective and cognitive processing of touch, oral texture, and temperature in the brain." Neuroscience \& Biobehavioral Reviews 34, no. 2 pp.237-245, 2010.

[15] L. A. Jones, and A. M. Smith. "Tactile sensory system: encoding from the periphery to the cortex." Wiley Interdisciplinary Reviews: Systems Biology and Medicine 6, no. 3 pp. 279-287, 2014.

[16] J. Fishel, V. J. Santos, and G. E. Loeb. "A robust micro-vibration sensor for biomimetic fingertips." in 2nd IEEE RAS \& EMBS International Conference on, pp. 659-663. IEEE, 2008.

[17] D. Reshef, Y. Reshef, H. Finucane, S. Grossman, G. McVean, P. Turnbaugh, E. Lander, M. Mitzenmacher, P. Sabeti. Detecting novel associations in large datasets. Science 334, 6062 (2011).

[18] L. Festinger, A theory of cognitive dissonance, Evanston, IL: Row \& Peterson, 1957.

[19] L. Festinger and J. M. Carlsmith, "Cognitive consequences of forced compliance. Journal of Abnormal and Social Psychology, 58, 203 - 210, 1959.

[20] A. Julio and E. Wästlund. "Exploring touch-screen biometrics for user identification on smart phones." In Privacy and Identity Management for Life, pp. 130-143. Springer Berlin Heidelberg, 2012.

[21] P. Rousseeuw, I. Ruts and J.W. Tukey, 'The bagplot: A bivariate boxplot', The American Statistician Vol. 53(4), pp.382-387, 1999.

[22] R.J. Hyndman, and S. H. Lin, "Rainbow plots, bagplots, and boxplots for functional data." Journal of Computational and Graphical Statistics 19, no. 1 (2010). 

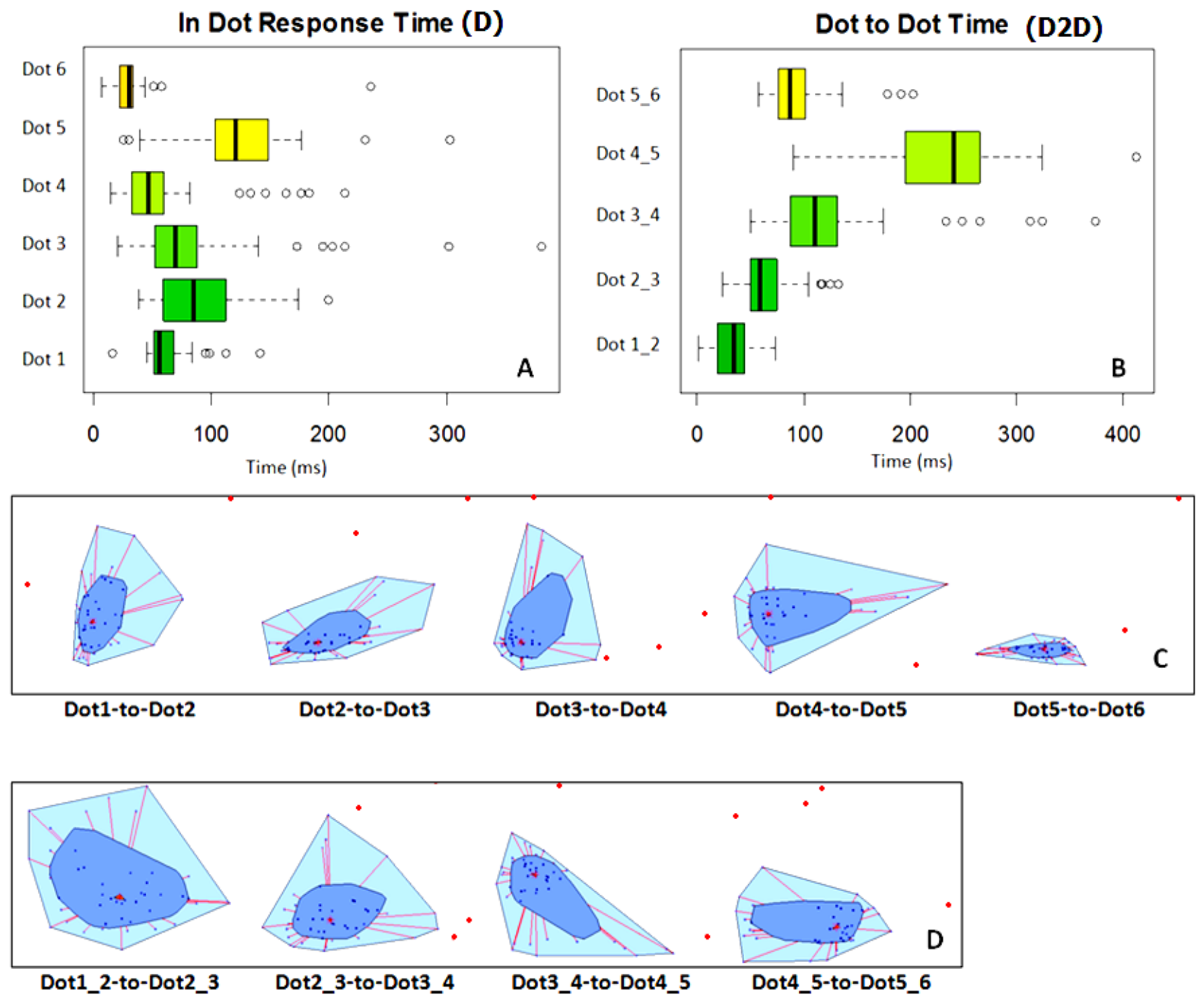

Fig. 8. Trends in Cognitive Consistency Analysis (Lock Pattern data): (A) Box plot of in-dot (D) response time; (B) Box plot of Dot-to-Dot time (D2D) and (c) Median and Outlier distributions in tapping on consecutive dots and (d) Median and Outlier distributions in consecutive dot-to-dot transitions 

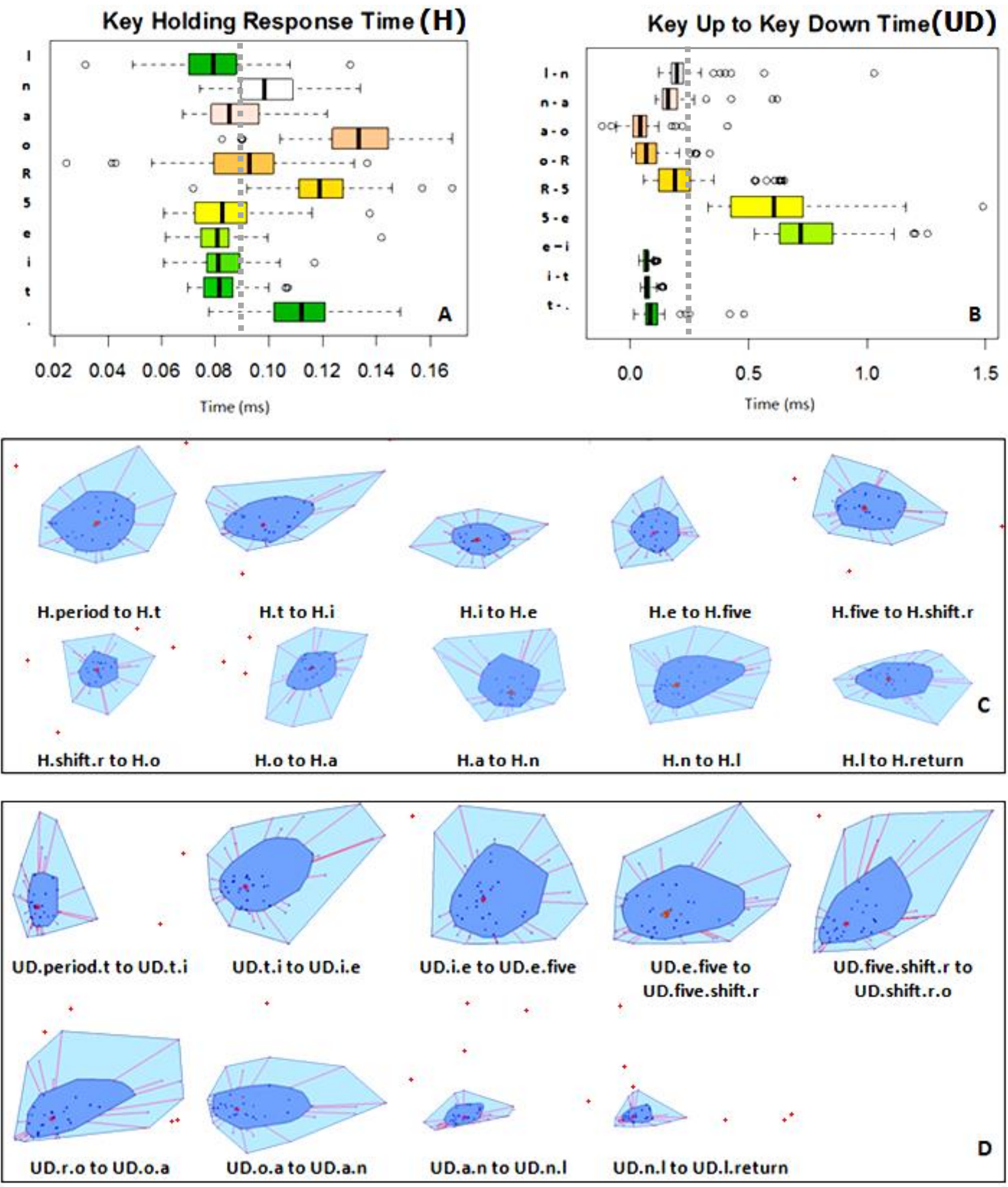

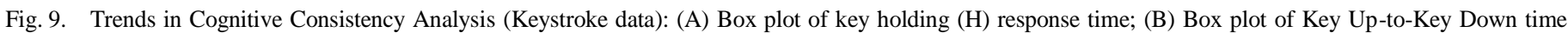
(UD) and (c) Median and Outlier distributions in consecutive key holding and (d) Median and Outlier distributions in consecutive key up to key down transitions 